\title{
Finite-time State Estimation for Delayed Neural Networks with Redundant Delayed Channels
}

\author{
Zhongyi Zhao, Zidong Wang, Lei Zou and Ge Guo
}

\begin{abstract}
The finite-time state estimation issue is addressed in this work for discrete time-delayed neural networks. More than one communication channel is utilized to improve the communication performance. The transmission delays of each channel are modeled by a family of stochastic variables which are independent and identically distributed. The main purpose of the current work is to construct an appropriate state estimation scheme under which the corresponding state estimation error dynamics is finite-time bounded in the mean square. By employing the stochastic analysis approach and introducing a special Lyapunov-like functional, we have developed certain sufficient conditions to achieve the prescribed estimation performance. Furthermore, the exact expressions of the achieved estimator parameters are given by solving a special minimization problem subject to certain inequality constraints. Finally, we propose an illustrative simulation to examine the correctness as well as the effectiveness of our proposed state estimation method.
\end{abstract}

Index Terms-Delayed neural networks, redundant delayed channels, state estimation, finite-time boundedness

\section{INTRODUCTION}

A tremendous amount of research interest have been witnessed in past several decades in neural networks (NNs) due primarily to their strong self-learning ability adapting to complex environment as well as their application potentials in multi-objective optimization and control issues. So far, plenty of successful applications of NNs have been found in various of practical areas including the automation control, pattern recognition, signal processing and optimization calculation [5], [11], [15], [27], [28], [35]. In recent several years, plenty of important research results have been derived on various analysis issues of the dynamic behaviors for NNs (such as stability, passivity analysis and synchronization), see e.g. [20][22], [30], [33]. Furthermore, It is worth pointing out that time delays would always exist in signal transmission for

This work was supported in part by the National Natural Science Foundation of China under Grants 61703245 and 61873148, the Taishan Scholar Project of Shandong Province of China, the China Postdoctoral Science Foundation under the Grant number 2016M600547, the Qingdao Postdoctoral Applied Research Project under the Grant number 2016117, the Postdoctoral Special Innovation Foundation of Shandong under the Grant number 201701015, the Royal Society of the U.K., and the Alexander von Humboldt Foundation of Germany. Corresponding author: Zidong Wang.

Z. Zhao and L. Zou are with the College of Electrical Engineering and Automation, Shandong University of Science and Technology, Qingdao 266590, China.

Z. Wang is with the College of Electrical Engineering and Automation, Shandong University of Science and Technology, Qingdao 266590, China. $\mathrm{He}$ is also with the Department of Computer Science, Brunel University London, Uxbridge, Middlesex, UB8 3PH, United Kingdom. (Email: Zidong.Wang@brunel.ac.uk)

G. Guo is with the Department of Automation, Dalian Maritime University, Dalian 116026, China. many artificial NNs and the effects of time delays might give rise to undesired oscillation and even the instability of the NNs. Accordingly, the dynamic behaviors of delayed NNs have been extensively considered and a significant number of valuable research results have appeared in the literature, see [7], [16], [23], [24], [31], [44]. Among others, a research topic that has been drawing particular research attention is the state estimation/filtering issue for NNs with various time delays (e.g. distributed, discrete and mixed delays).

As a hot research topic in signal processing and control areas, state estimation/filtering issue has gained significant research interest due primarily to their wide application in industry [2], [29]. For NNs, the state information is always necessary for dealing with certain tasks including optimization and control. Unfortunately, the state information of NNs might not be always fully available (or accessible) due to a number of reasons (e.g. the large network size and the resource constraints). As such, the state estimation problem from available network measurements becomes critically important for successful utilization of NNs in engineering practice. By now, different state estimation schemes have been developed which include, but are not limited to, the well-known Kalman filtering [13], [18], the $\mathcal{H}_{\infty}$ state estimation [9], [41] and the set-membership state estimation [10], [42] approaches. To mention just a few, the Kalman filtering technique has been recognized as a credible estimation method to deal with linear systems with Gaussian noises, but it might lead to unsatisfactory performance if the external noises are not strictly Gaussian. For the system with energy-bounded noises, the $\mathcal{H}_{\infty}$ state estimation is an ideal estimation scheme which aims to provide a fixed disturbance attenuation level on the state estimation error. Furthermore, the set-membership state estimation method is able to handle the estimation task for systems with unknown-but-bounded noises, which guarantees that the SEE is confined to certain ellipsoid at each time step.

In most existing results concerning the state estimation issues, the asymptotic (or exponential) stability is the main concern that represents a type of steady-state behavior defined in the infinite-time horizon [3], [8]-[10], [12], [37], [41], [42]. Transient properties, on the other hand, are vitally important as well for some engineering applications. In many practical systems, it is always required that the system could achieve certain transient properties (e.g. finite-time convergence) over a finite horizon with guaranteed steady-state properties, and therefore it makes practical sense to study the transient behaviors over the finite time interval. Consequently, the so-called finite-time stability (FTS), finite-time boundedness (FTB) and finite-time tracking (FTT) have recently attracted quite a lot 
This article has been accepted for publication in a future issue of this journal, but has not been fully edited. Content may change prior to final publication. Citation information: DOI10.1109/TSMC.2018.2874508, IIEEE Transactions on Systems, Man, and Cybernetics: Systems

of research attention [13], [34], [38]-[40]. Compared with the well-investigated FTS, the aim of the FTB is to ensure that the state trajectory could reach a bounded set within certain given finite time. It is worth mentioning that, in practical engineering, FTB is sometimes more reasonable since the stability might be difficult to achieve due to various reasons such as persistently bounded disturbances. Up to now, various FTB control/estimation problems have started to attract some initial research interest, see e.g. [34], [36], [39]. For instance, in [39], the finite-time state estimation (FTSE) problem for recurrent delayed NNs subject to component-based eventtriggered communication has been examined. In order to quantify the estimation performance, a special performance named finite-time bounded in the mean square (FTBMS) has been proposed in [39] where the desired estimator parameter has been obtained through solving a constrained optimization problem. In [36], the $\mathcal{H}_{\infty}$ control issue has been addressed for a certain type of Markovian jump systems subject to the average dwell time switching, in which the time-varying transition probability is partly unknown. Sufficient conditions for the FTB of the concerned Markovian jump system have been derived under which the system trajectory is enforced to stay within a prescribed bound.

In response to the rapid development of networked communication, more and more signal transmissions are implemented via the communication network. As such, increasing research efforts have been devoted to the analysis and synthesis problems with different network-induced effects including network-induced delays, signal quantization, channel fading, packet dropouts [1], [6], [14], [17], [43], [45]. For example, in [6], partly known distribution transmission delays have been considered and the corresponding $\mathcal{H}_{\infty}$ filtering issue of networked systems has been studied. For artificial NNs, it is often the case that we are only able to acquire the observations (e.g. the measurement data) transmitted via network channels (e.g. communication networks with limited bandwidth) with certain communication constraints. As such, the state estimation problems for NNs with network-based communication have recently gained particular research attention. In order to enhance the reliability of transmitted information, a novel network-based communication scheme called redundant channel communication has been employed in [14] to cope with the $\mathcal{H}_{\infty}$ state estimation issue. In such a communication scheme, one more channel is adopted as a redundant one aiming to reduce the possibility of packet losses in the single-channel case. In [43], the distributed $\mathcal{H}_{\infty}$ filtering problem with redundant channels has been addressed for a type of Markov jump Lur'e systems subject to stochastic switching topologies over sensor networks. Generally speaking, communication over redundant channels is a good scheme to improve the communication performance since more information could be employed for estimation tasks as compared with the signal channel. It is worth mentioning that, current research works about the redundant channels have only considered redundant channels with packet dropouts. As far as the authors' knowledge goes, the research on the redundant channels with transmission delays has not yet been fully studied despite its explicit practical insight in communication and control areas. This leads to the primary motivation of our study.

According to the above discussions, in this work, we shall consider the FTSE problem for a type of delayed NNs with redundant delayed channels. This is a non-trivial task because of the following two identified difficulties: 1) how to design the state estimator for the considered neural networks under the effects of redundant delayed channels? and 2) how to achieve the desired estimator parameters to guarantee that the desired finite-time performance requirement is satisfied? The main purpose of this paper to provide satisfactory answers to these two questions. Following are the primary contributions of the current work. (1) The state estimation issue is, for the first time, investigated for NNs subject to delayed redundant channels. (2) A novel SE is developed for dealing with the finite-time state estimation (FTSE) issue. (3) An optimization problem is addressed to achieve the desired estimator parameters by minimizing the settling-like time (SLT).

The remaining parts of this work are summarized as follows. The mathematical model of our considered problem is presented in Section II including the mechanism of redundant delayed channels. Then, in Section III, we achieve sufficient conditions to deal with the FTB problem for the state estimation error (SEE) in the mean square by solving a special constrained optimization issue. Section IV provide an illustrative simulation example to confirm the correctness as well as the effectiveness of the developed estimation method. Section V is a summary of this paper.

Notation: The notation utilized in this work is quite standard. In this work, $\mathbb{Z}^{+}$stands for the set of all nonnegative integers. $\mathbb{R}^{n \times m}$ denotes the set of all $n \times m$ real-valued matrices. $\mathbb{R}^{n}$ represents the $n$-dimensional Euclidean space. If the dimension of a matrix is not specified, it means that the matrix has compatible dimension. The superscript " $T$ " denotes matrix transposition. 0 and $I$ denotes, respectively, the zero matrix and identity matrix with appropriate dimensions. The notation $S \leq 0$ (respectively, $S<0$ ) denotes that $S$ is a real symmetric and negative semi-definite (respectively, negative definite) matrix. The notation $\|F\|$ denotes the usual Euclidean norm of vector $F$. The asterisk "*” is utilized to denote a term which is induced by symmetry in symmetric block matrices. $\mathbb{E}\{x\}$ denotes expectation of $x$. The block-diagonal matrix is denoted by $\operatorname{diag}\{\ldots\} \cdot \operatorname{diag}_{n}\{X\}$ represents the special block-diagonal matrix with the same block $X$ (i.e. $\operatorname{diag}\{\underbrace{X, X, \cdots, X}_{n}\}$ ). $\operatorname{vec}_{n}\left\{X_{i}\right\}$ denotes $\left[\begin{array}{llll}X_{1}^{T} & X_{2}^{T} & \cdots & X_{n}^{T}\end{array}\right]^{T}$. $\lceil$.$\rceil denotes top$ integral function. $\delta$ denotes Kronecker delta function of which definition is $\delta(i)=\left\{\begin{array}{ll}1, & i=0 \\ 0, & i \neq 0\end{array}\right.$.

\section{Preliminaries and Problem Statement}

In this work, we consider a special type of delayed discretetime NNs with noise disturbance of the following form:

$$
\left\{\begin{aligned}
x(k+1)= & A x(k)+F f(x(k))+B \omega(k) \\
& +G g(x(k-d(k)))+J(k) \\
y(k)= & C x(k) \\
z(k)= & L x(k) \\
x(k)= & \phi(k), \quad 0 \geq k \geq-\max \{d(k)\}
\end{aligned}\right.
$$


where $x(k) \in \mathbb{R}^{n_{x}}$ is the neural state vector; $f(x(k))=$ $\operatorname{vec}_{n_{x}}\left\{f_{i}\left(x_{i}(k)\right)\right\}$ and $g(x(k))=\operatorname{vec}_{n_{x}}\left\{g_{i}\left(x_{i}(k)\right)\right\}$ denote the nonlinear activation function; $\omega(k)$ stands for a Gaussian noise satisfying the conditions $\mathbb{E}\left\{\omega^{2}(k)\right\}=1$ and $\mathbb{E}\{\omega(k)\}=0$; $J(k) \in \mathbb{R}^{n_{x}}$ is the external bias; $y(k) \in \mathbb{R}^{n_{y}}$ represents the output of the neurons; $z(k) \in \mathbb{R}^{n_{z}}$ means the signal to be estimated; $\phi(k)$ represents the initial condition. $A=$ $\operatorname{diag}\left\{a_{1}, a_{2}, \cdots, a_{n_{x}}\right\}$ denotes the state feedback coefficient matrix; $G$ and $F$ denote, respectively, the delayed connection weight matrix and the connection weight matrix; $B, C$ and $L$ are the known real-valued matrices; $d(k)$ characterizes the time-varying discrete time delay.

Assumption 1: For any given positive constant $k$, the timedelay $d(k)$ satisfies

$$
d_{m} \leq d(k) \leq d_{M}
$$

in which $d_{m}$ and $d_{M}$ are the known nonnegative integers.

Assumption 2: [25] For any $t, s \in \mathbb{R}, s \neq t$, the nonlinear functions $g$ and $f$ in (1) satisfy $g(0)=0, f(0)=0$ and

$$
\begin{gathered}
l_{i}^{-} \leq \frac{f_{i}(s)-f_{i}(t)}{s-t} \leq l_{i}^{+} ; i=1,2, \cdots, n_{x} \\
m_{i}^{-} \leq \frac{g_{i}(s)-g_{i}(t)}{s-t} \leq m_{i}^{+} ; i=1,2, \cdots, n_{x}
\end{gathered}
$$

where $l_{i}^{-}, l_{i}^{+}, m_{i}^{-}, m_{i}^{+}$denote some known scalars.

Remark 1: As is shown in [25], the scalars $l_{i}^{+}, l_{i}^{1}, m_{i}^{+}, m_{i}^{1}$ in Assumption 2 could be zero, negative or positive. As such, it is clear that the nonlinear activation functions are allowed to be non-monotonic, and these functions are more general compared with the usual sigmoid functions.

In order to enhance the communication reliability, in this work, redundant communication channels are utilized to deal with the data transmission between the $\mathrm{NN}$ and the remote SE. Without loss of generality, it is supposed that there are $l$ communication channels adopted between the $\mathrm{NN}$ and the estimator, which are specifically shown in the Fig.1. We consider the case that the transmission delay would occur in each communication channel. Let the output signal transmitted via the $s$-th channel be denoted by $\bar{y}_{s}(k)(s \in\{1,2, \cdots, l\})$. Obviously, $\bar{y}_{s}(k)$ could be written as follows:

$$
\bar{y}_{s}(k)=y\left(k-\tau_{s}(k)\right)+D_{s} v_{s}(k)
$$

in which $v_{s}(k)$ represents a Gaussian white noise of the $s$-th channel satisfying

$$
\begin{aligned}
\mathbb{E}\left\{v_{i}\right\} & =0, i=1,2, \cdots, l \\
\mathbb{E}\left\{v_{i} v_{j}\right\} & =\left\{\begin{array}{l}
1, i=j \\
0, i \neq j
\end{array}\right.
\end{aligned}
$$

$\tau_{s}(k)\left(k \in \mathbb{Z}^{+}, s=1,2, \ldots, l\right)$ denotes the transmission delay of the $s$-th channel which is assumed to be a sequence of independent identically distributed random variables. Let $\tau_{s}(k) \in S \triangleq\{0,1, \ldots, \bar{\tau}\}$ for all the $k$ and $s$. Furthermore, the occurrence probability of $\tau_{s}(k)=t(t \in S)$ is given by

$$
\operatorname{Pr}\left\{\tau_{s}(k)=t\right\}=p_{s t}, s=1,2, \cdots, l ; t=0,1, \cdots, \bar{\tau}
$$

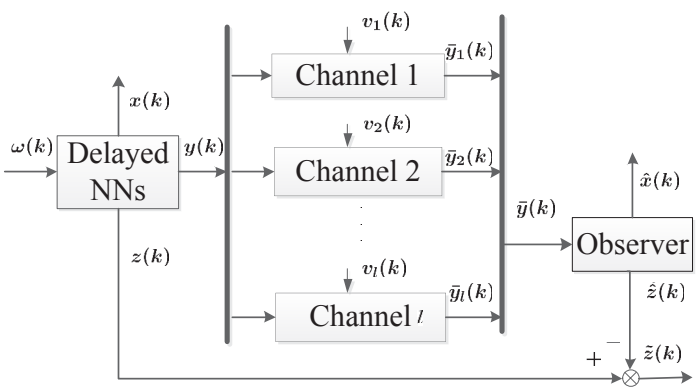

Fig. 1: The estimation scheme with redundant delayed channels.

Then, denoting $\bar{k}_{t}=k-t$ and using the Kronecker delta function, $\bar{y}_{s}(k)$ can be reformulated as follows

$$
\bar{y}_{s}(k)=\sum_{t=0}^{\bar{\tau}} \delta\left(t-\tau_{s}(k)\right) C x\left(\bar{k}_{t}\right)+D_{s} v_{s}(k)
$$

Obviously, the output signal $\bar{y}_{s}(k)$ contains both the "distributed delays" and "random variables", which gives rise to the main difficulty in designing the estimator based on the received signal $\bar{y}(k) \triangleq\left[\begin{array}{lllll}\bar{y}_{1}^{T}(k) & \bar{y}_{2}^{T}(k) & \cdots & \bar{y}_{l}^{T}(k)\end{array}\right]^{T}$.

Remark 2: It is easy to see from the developed measurement model (4) and the probability distribution (7) that the redundant channels could transmit more information than single channel. More specifically, it could be found that the probability of $\bar{y}_{s}(k)=y\left(\bar{k}_{t}\right)+D_{s} v_{s}(k)$ is $p_{s t}$. If only one communication channel is utilized (e.g. only channel 1 is employed), the probability that $\bar{y}(k)$ contains the information about $y\left(\bar{k}_{t}\right)$ is $p_{1 t}$. However, if $l$ communication channels are adopted to transmit data, the probability that $\bar{y}(k)$ contains the information about $y\left(\bar{k}_{t}\right)$ is $\sum_{i=1}^{l} p_{i t}$, which is larger than the single-channel case. In other words, as the number of redundant delayed channels increases, the probability of $\bar{y}(k)$ including the information of delayed signal $y_{i}\left(\bar{k}_{0}\right), y_{i}\left(\bar{k}_{1}\right), \cdots, y_{i}\left(\bar{k}_{\bar{\tau}}\right), i=1,2, \ldots, l$ would increase, and this enables us to retrieve more useful information. In other words, redundant channels could largely enhance the communication performance between the $\mathrm{NN}$ and the remote SE.

To achieve the estimates of the states for the NNs (1), we develop a SE with the following form:

$$
\left\{\begin{aligned}
\hat{x}(k+1)= & A \hat{x}(k)+F f(\hat{x}(k))+G g(\hat{x}(k-d(k))) \\
& +J(k)+\sum_{s=1}^{l}\left(K _ { s } \left(\bar{y}_{s}(k)-\sum_{t=0}^{\bar{\tau}} \delta\left(t-\tau_{s}(k)\right)\right.\right. \\
& \left.\left.\times C \hat{x}\left(\bar{k}_{t}\right)\right)\right) \\
\hat{z}(k)= & L \hat{x}(k) \\
\hat{x}(k)= & \hat{\phi}(k), \quad 0 \geq k \geq-d_{M}
\end{aligned}\right.
$$

where $\hat{x}(k) \in \mathbb{R}^{n_{x}}$ stands for the estimate of the state $x(k)$ and $\hat{z}(k)$ means the corresponding estimate of the signal $z(k)$; $\hat{\phi}(k)$ is the initial condition; and $K_{1}, K_{2}, \ldots, K_{l}$ stand for the estimator gain matrices that need to be determined. Then, letting $\tilde{z}(k)=z(k)-\hat{z}(k)$ and $e(k)=x(k)-\hat{x}(k)$, we could 
obtain the estimation dynamics according to (1) and (9) as follows:

$$
\left\{\begin{aligned}
e(k+1)= & A e(k)+F \tilde{f}(e(k))+G \tilde{g}(e(k-d(k))) \\
& -\sum_{s=1}^{l}\left(K_{s} \sum_{t=0}^{\bar{\tau}} \delta\left(t-\tau_{s}(k)\right) C e\left(\bar{k}_{t}\right)\right) \\
& +B \omega(k)-\sum_{s=1}^{l} K_{s} D_{s} v_{s}(k) \\
\tilde{z}(k)= & L e(k)
\end{aligned}\right.
$$

in which

$$
\begin{aligned}
& \tilde{g}(e(k)) \triangleq g(x(k))-g(\hat{x}(k)), \\
& \tilde{f}(e(k)) \triangleq f(x(k))-f(\hat{x}(k)) .
\end{aligned}
$$

Definition 1: [34] Assume that there exist a time-based function $k_{*}$ satisfying the following condition:

$$
\mathbb{E}\left\{\|\tilde{z}(k)\|^{2}\right\} \leq \varepsilon_{*}, \forall k \geq k_{*}
$$

where $k_{*}=k_{*}\left(e(0), \varepsilon_{*}\right)$ is the SLT function and $\varepsilon_{*}>0$ is a given upper bound. Then, the dynamical system (10) is FTBMS.

The purpose of our research is to handle the FTSE issue for a type of discrete time-delayed NNs (1). Specifically, our main attention would be focused on the design issue of the estimator parameter gains $K_{1}, K_{2}, \cdots, K_{l}$ such that the SEE dynamics (10) is FTBMS with minimized SLT $k_{*}$.

\section{Main Results}

We firstly achieve the sufficient condition in this Section to ensure that the SEE dynamics (10) is FTBMS. Before giving the main results of our work, let us introduce some necessary lemmas.

Lemma 1: [4] Letting the matrices $Y_{1}, Y_{2}, Y_{3}$ be given in which $Y_{1}=Y_{1}^{T}$ and $Y_{2}=Y_{2}^{T}>0$, then $Y_{1}+Y_{3}^{T} Y_{2}^{-1} Y_{3}<0$ if and only if

$$
\left[\begin{array}{cc}
Y_{1} & Y_{3}^{T} \\
Y_{3} & -Y_{2}
\end{array}\right]<0, \quad \text { or } \quad\left[\begin{array}{cc}
-Y_{2} & Y_{3} \\
Y_{3}^{T} & Y_{1}
\end{array}\right]<0
$$

Lemma 2: [25] Let $\eta=\left[\eta_{1}, \eta_{2}, \cdots, \eta_{n}\right]^{T} \in \mathbb{R}^{n}$ and $f(\eta)=\left[f_{1}\left(\eta_{1}\right), f_{2}\left(\eta_{2}\right), \cdots, f_{n}\left(\eta_{n}\right)\right]^{T} \in \mathbb{R}^{n}$ be a continuous nonlinear function satisfying $\iota_{p}^{-} \leq \frac{f_{i}(\epsilon)}{\epsilon} \leq \iota_{p}^{+}, \epsilon \neq 0, \epsilon \in$ $\mathbb{R}, 1 \leq p \leq n$ with $\iota_{p}^{-}$and $\iota_{p}^{+}$being known scalars. Suppose that $\Lambda=\operatorname{diag}\left(\lambda_{1}, \lambda_{2}, \cdots, \lambda_{n}\right)$ is positive semi-definite. Then

$$
\left[\begin{array}{c}
\eta \\
f(\eta)
\end{array}\right]^{T}\left[\begin{array}{cc}
\Lambda M_{1} & -\Lambda M_{2} \\
-\Lambda M_{2} & \Lambda
\end{array}\right]\left[\begin{array}{c}
\eta \\
f(\eta)
\end{array}\right] \leq 0
$$

where $M_{1}=\operatorname{diag}\left(\vec{\iota}_{1}, \vec{\iota}_{2}, \cdots, \vec{\iota}_{n}\right), M_{2}=\operatorname{diag}\left(\bar{\iota}_{1}, \bar{\iota}_{2}, \cdots, \bar{\iota}_{n}\right)$, $\overrightarrow{\iota_{i}}=\iota_{i}^{+} \iota_{i}^{-}$and $\vec{\iota}_{i}=\iota_{i}^{-}+\iota_{i}^{+}$.

Theorem 1: Consider the estimator error dynamics (10) and let the positive scalar $0<\gamma<1$, estimator gain matrices $K_{1}, K_{2}, \ldots, K_{l}$ and the desired upper bound of SEE $\varepsilon_{*}$ be given. Then, the dynamical system (10) is FTBMS if there exist $l+4$ positive definite matrices (PDMs) $Q>0, P>0$, $R_{i}>0(i=1,2, \cdots, l), \Lambda \triangleq \operatorname{diag}\left(\lambda_{1}, \lambda_{2}, \cdots, \lambda_{n_{x}}\right)>0$, $\Gamma \triangleq \operatorname{diag}\left(\gamma_{1}, \gamma_{2}, \cdots, \gamma_{n_{x}}\right)>0$ satisfying

$$
\Phi<0
$$

$$
\begin{aligned}
L^{T} L & \leq P \\
\theta & <(1-\gamma) \varepsilon_{*}
\end{aligned}
$$

where $\bar{\tau}_{i}=\bar{\tau}+i$ and

$$
\begin{aligned}
& \Omega_{11}=\left[\begin{array}{cccc}
\Phi_{00} & \Phi_{01} & \cdots & \Phi_{0 \bar{\tau}} \\
* & \Phi_{11} & \cdots & \Phi_{1 \bar{\tau}} \\
* & * & \ddots & \vdots \\
* & * & * & \Phi_{\bar{\tau} \bar{\tau}}
\end{array}\right], \Phi=\left[\begin{array}{cc}
\Omega_{11} & \Omega_{12} \\
* & \Omega_{22}
\end{array}\right], \\
& \Omega_{12}=\left[\begin{array}{lll}
\Phi_{0, \bar{\tau}_{1}} & \Phi_{0, \bar{\tau}_{2}} & \Phi_{0, \bar{\tau}_{3}} \\
\urcorner_{i, \bar{\tau}_{1}} & \urcorner_{i, \bar{\tau}_{2}} & \urcorner_{i, \bar{\tau}_{3}}
\end{array}\right], \\
& \rceil_{i, \bar{\tau}+j}=\operatorname{vec}_{\bar{\tau}}\left\{\Phi_{i, \bar{\tau}_{j}}\right\}, \quad j=1,2,3, \\
& \Omega_{22}=\left[\begin{array}{ccc}
\Phi_{\bar{\tau}_{1}, \bar{\tau}_{1}} & \Phi_{\bar{\tau}_{1}, \bar{\tau}_{2}} & \Phi_{\bar{\tau}_{1}, \bar{\tau}_{3}} \\
* & \Phi_{\bar{\tau}_{2}, \bar{\tau}_{2}} & \Phi_{\bar{\tau}_{2}, \bar{\tau}_{3}} \\
* & * & \Phi_{\bar{\tau}_{3}, \bar{\tau}_{3}}
\end{array}\right], \\
& \Phi_{00}=A^{T} P A+\sum_{i=1}^{l} \bar{\tau} R_{i}-\gamma P-\Lambda L_{1}-\Gamma M_{1} \\
& -\sum_{i=1}^{l} p_{i 0} A^{T} P K_{i} C-\sum_{i=1}^{l} p_{i 0} C^{T} K_{i}^{T} P A \\
& +\sum_{i=1}^{l} p_{i 0} C^{T} K_{i}^{T} P K_{i} C \\
& +2 \sum_{1 \leq i<j \leq l} p_{i 0} p_{j 0} C^{T} K_{i}^{T} P K_{j} C \\
& \Phi_{s t}=-\sum_{i=1}^{l} \gamma^{s} R_{i}+\sum_{i=1}^{l} p_{i s} C^{T} K_{i}^{T} P K_{i} C \\
& +2 \sum_{1 \leq i<j \leq l} p_{i s} p_{j t} C^{T} K_{i}^{T} P K_{j} C, \\
& s=t \in\{1,2, \cdots, \bar{\tau}\} \\
& \Phi_{s t}=\sum_{1 \leq i<j \leq l} p_{i s} p_{j t} C^{T} K_{i}^{T} P K_{j} C-\sum_{i=1}^{l} p_{i t} A^{T} P K_{i} C \\
& +\sum_{1 \leq i<j \leq l} p_{i t} p_{j s} C^{T} K_{j}^{T} P K_{i} C, \\
& s=0, t=1,2, \cdots, \bar{\tau} \\
& \Phi_{s t}=\sum_{1 \leq i<j \leq l} p_{i s} p_{j t} C^{T} K_{i}^{T} P K_{j} C \\
& +\sum_{1 \leq i<j \leq l} p_{i t} p_{j s} C^{T} K_{j}^{T} P K_{i} C, \\
& 1 \leq s<t \leq \bar{\tau} \\
& \Phi_{i, \bar{\tau}_{1}}=\left\{\begin{array}{l}
A^{T} P F-\sum_{s=1}^{l} p_{s i} C^{T} K_{s}^{T} P F+\Lambda L_{2}, \quad i=0 \\
-\sum_{s=1}^{l} p_{s i} C^{T} K_{s}^{T} P F, \quad i=1,2, \cdots, \bar{\tau}
\end{array}\right. \\
& \Phi_{i, \bar{\tau}_{2}}=\left\{\begin{array}{l}
\Gamma M_{2}, \quad i=0 \\
0, \quad i=1,2, \cdots, \bar{\tau}
\end{array}\right.
\end{aligned}
$$




$$
\begin{aligned}
& \Phi_{i, \bar{\tau}_{3}}=\left\{\begin{array}{l}
A^{T} P G-\sum_{s=1}^{l} p_{s i} C^{T} K_{s}^{T} P G, i=0 \\
-\sum_{s=1}^{l} p_{s i} C^{T} K_{s}^{T} P G, \quad 1 \leq i \leq \bar{\tau}
\end{array}\right. \\
& \Phi_{\bar{\tau}_{1}, \bar{\tau}_{1}}=-\Lambda+F^{T} P F, \Phi_{\bar{\tau}_{1}, \bar{\tau}_{2}}=0, \\
& \Phi_{\bar{\tau}_{1}, \bar{\tau}_{3}}=F^{T} P G, \Phi_{\bar{\tau}_{2}, \bar{\tau}_{2}}=\left(d_{M}-d_{m}+1\right) Q-\Gamma, \\
& \Phi_{\bar{\tau}_{2}, \bar{\tau}_{3}}=0, \Phi_{\bar{\tau}_{3}, \bar{\tau}_{3}}=G^{T} P G-Q, \\
& \hat{l}_{p}=l_{p}^{+} l_{p}^{-}, \widehat{l}_{p}=\frac{l_{p}^{+}+l_{p}^{-}}{2}, \hat{m}_{p}=m_{p}^{+} m_{p}^{-}, \\
& \widehat{m}_{p}=\frac{m_{p}^{+}+m_{p}^{-}}{2}, p=1,2, \cdots, n_{x}, \\
& L_{1}=\operatorname{diag}\left(\hat{l}_{1}, \hat{l}_{2}, \cdots, \hat{l}_{n_{x}}\right), M_{1}=\operatorname{diag}\left(\hat{m}_{1}, \hat{m}_{2}, \cdots, \hat{m}_{n_{x}}\right), \\
& L_{2}=\operatorname{diag}\left(\widehat{l}_{1}, \widehat{l}_{2}, \cdots, \widehat{l}_{n_{x}}\right), M_{2}=\operatorname{diag}\left(\widehat{m}_{1}, \widehat{m}_{2}, \cdots, \widehat{m}_{n_{x}}\right), \\
& \theta=B^{T} P B+\sum_{s=1}^{l} D_{s}^{T} K_{s}^{T} P K_{s} D_{s} .
\end{aligned}
$$

In addition, if the condition mentioned above is satisfied, the SLT $k_{*}$ can be calculated by

$$
k_{*}=\left\{\begin{array}{c}
0, \varepsilon_{*} \geq \frac{\mathbb{E}\{V(0)\}}{1-\sigma} \\
\left\lceil\log _{\gamma} \frac{(1-\sigma) \varepsilon_{*}}{\mathbb{E}\{V(0)\}}\right\rceil, \varepsilon_{*}<\frac{\mathbb{E}\{V(0)\}}{1-\sigma}
\end{array}\right.
$$

where $\sigma \in(0,1)$ is a scalar satisfying

$$
\frac{1}{1-\gamma}\left(\sum_{i=1}^{l} D_{i}^{T} K_{i}^{T} P K_{i} D_{i}+B^{T} P B\right)=\sigma \varepsilon_{*}
$$

Proof: Choose a Lyapunov-like functional candidate of the following form:

$$
\mathcal{V}(k)=\sum_{j=1}^{4} \mathcal{V}_{j}(k)
$$

where

$$
\begin{aligned}
& \mathcal{V}_{1}(k)=e^{T}(k) P e(k) \\
& \mathcal{V}_{2}(k)=\sum_{j=-d(k)+k}^{-1+k} \gamma^{k-1-j} \tilde{g}^{T}(e(j)) Q \tilde{g}(e(j)) \\
& \mathcal{V}_{3}(k)=\sum_{i=d_{m}}^{d_{M}-1} \sum_{j=-i+k}^{-1+k} \gamma^{k-1-j} \tilde{g}^{T}(e(j)) Q \tilde{g}(e(j)) \\
& \mathcal{V}_{4}(k)=\sum_{s=1}^{l} \sum_{t=1}^{\bar{\tau}} \sum_{j=-t+k}^{-1+k} \gamma^{k-1-j} e^{T}(j) R_{s} e(j)
\end{aligned}
$$

From (10), one obtains that

$$
\begin{aligned}
& \mathbb{E}\left\{(1-\gamma) \mathcal{V}_{1}(k)+\Delta \mathcal{V}_{1}(k)\right\} \\
= & \mathbb{E}\left\{\mathcal{V}_{1}(k+1)-\gamma \mathcal{V}_{1}(k)\right\} \\
= & \mathbb{E}\left\{e^{T}(k+1) P e(k+1)-\gamma e^{T}(k) P e(k)\right\} \\
= & \mathbb{E}\left\{e^{T}(k)\left(A^{T} P A-\gamma P\right) e(k)\right. \\
& +2 e^{T}(k) A^{T} P F \tilde{f}(e(k)) \\
& +2 e^{T}(k) A^{T} P G \tilde{g}(e(k-d(k)))
\end{aligned}
$$

$$
\begin{aligned}
& -2 \sum_{s=1}^{l} \sum_{t=0}^{\bar{\tau}} p_{s t} e^{T}(k) A^{T} P K_{s} C e\left(\bar{k}_{t}\right) \\
& +\tilde{f}^{T}(e(k)) F^{T} P F \tilde{f}(e(k)) \\
& +2 \tilde{f}^{T}(e(k)) F^{T} P G \tilde{g}(e(k-d(k))) \\
& -2 \sum_{s=1}^{l} \sum_{t=0}^{\bar{\tau}} p_{s t} \tilde{f}^{T}(e(k)) F^{T} P K_{s} C e\left(\bar{k}_{t}\right) \\
& +\tilde{g}^{T}(e(k-d(k))) G^{T} P G \tilde{g}(e(k-d(k))) \\
& -2 \sum_{s=1}^{l} \sum_{t=0}^{\bar{\tau}} p_{s t} \tilde{g}^{T}(e(k-d(k))) G^{T} P K_{s} C e\left(\bar{k}_{t}\right) \\
& +\sum_{s=1}^{l} \sum_{t=0}^{\bar{\tau}} p_{s t} e^{T}\left(\bar{k}_{t}\right) C^{T} K_{s}^{T} P K_{s} C e\left(\bar{k}_{t}\right) \\
& +2 \sum_{1 \leq i<j \leq l} \sum_{s=0}^{\bar{\tau}} \sum_{t=0}^{\bar{\tau}} p_{i s} p_{j t} \\
& \times e^{T}\left(\bar{k}_{s}\right) C^{T} K_{i}^{T} P K_{j} C e\left(\bar{k}_{t}\right) \\
& \left.+B^{T} P B+\sum_{s=1}^{l} D_{s}^{T} K_{s}^{T} P K_{s} D_{s}\right\}
\end{aligned}
$$

$$
\begin{aligned}
& \mathbb{E}\left\{(1-\gamma) \mathcal{V}_{2}(k)+\Delta \mathcal{V}_{2}(k)\right\} \\
= & \mathbb{E}\left\{\sum_{j=-d(k+1)+k}^{k} \gamma^{k-j} \tilde{g}^{T}(e(j)) Q \tilde{g}(e(j))\right. \\
& \left.-\gamma \sum_{j=k-d(k)}^{-1+k} \gamma^{k-1-j} \tilde{g}^{T}(e(j)) Q \tilde{g}(e(j))\right\} \\
\leq & \mathbb{E}\left\{\sum_{j=k-d_{M}+1}^{k-d_{m}} \gamma^{k-j} \tilde{g}^{T}(e(j)) Q \tilde{g}(e(j))-\tilde{g}^{T}(e(k-d(k))) Q\right. \\
& \left.\times \tilde{g}(e(k-d(k)))+\tilde{g}^{T}(e(k)) Q \tilde{g}(e(k))\right\}
\end{aligned}
$$

$$
\begin{aligned}
& \mathbb{E}\left\{(1-\gamma) \mathcal{V}_{3}(k)+\Delta \mathcal{V}_{3}(k)\right\} \\
= & \mathbb{E}\left\{\sum_{i=d_{m}}^{-1+d_{M}} \sum_{j=-i+k+1}^{k} \gamma^{k-j} \tilde{g}^{T}(e(j)) Q \tilde{g}(e(j))\right. \\
& \left.-\gamma \sum_{i=d_{m}}^{-1+d_{M}} \sum_{j=-i+k}^{-1+k} \gamma^{-j+k-1} \tilde{g}^{T}(e(j)) Q \tilde{g}(e(j))\right\} \\
= & \mathbb{E} \sum_{i=d_{m}}^{d_{M}-1}\left\{\tilde{g}^{T}(e(k)) Q \tilde{g}(e(k))\right. \\
& \left.-\gamma^{i} \tilde{g}^{T}\left(e\left(\bar{k}_{i}\right)\right) Q \tilde{g}\left(e\left(\bar{k}_{i}\right)\right)\right\} \\
= & \mathbb{E}\left\{\left(d_{M}-d_{m}\right) \tilde{g}^{T}(e(k)) Q \tilde{g}(e(k))\right. \\
& \left.-\sum_{i=d_{m}}^{d_{M}-1} \gamma^{i} \tilde{g}^{T}\left(e\left(\bar{k}_{i}\right)\right) Q \tilde{g}\left(e\left(\bar{k}_{i}\right)\right)\right\} \\
= & \mathbb{E}\left\{\left(d_{M}-d_{m}\right) \tilde{g}^{T}(e(k)) Q \tilde{g}(e(k))\right.
\end{aligned}
$$




$$
\begin{aligned}
- & \left.\sum_{j=k-d_{M}+1}^{k-d_{m}} \gamma^{k-j} \tilde{g}^{T}(e(j)) Q \tilde{g}(e(j))\right\} \\
& \mathbb{E}\left\{(1-\gamma) \mathcal{V}_{4}(k)+\Delta \mathcal{V}_{4}(k)\right\} \\
= & \mathbb{E}\left\{\sum_{s=1}^{l} \sum_{t=1}^{\bar{\tau}} \sum_{j=-t+k+1}^{k} \gamma^{\bar{k}_{j}} e^{T}(j) R_{s} e(j)\right. \\
& \left.-\gamma \sum_{s=1}^{l} \sum_{t=1}^{\bar{\tau}} \sum_{j=-t+k}^{-1+k} \gamma^{-j+k-1} e^{T}(j) R_{s} e(j)\right\} \\
= & \mathbb{E}\left\{\sum_{s=1}^{l} \sum_{t=1}^{\bar{\tau}} e^{T}(k) R_{s} e(k)\right. \\
& \left.-\sum_{s=1}^{l} \sum_{t=1}^{\bar{\tau}} \gamma^{t} e^{T}\left(\bar{k}_{t}\right) R_{s} e\left(\bar{k}_{t}\right)\right\} \\
= & \mathbb{E}\left\{\sum_{s=1}^{l} \bar{\tau} e^{T}(k) R_{s} e(k)\right. \\
& \left.-\sum_{s=1}^{l} \sum_{t=1}^{\bar{\tau}} \gamma^{t} e^{T}\left(\bar{k}_{t}\right) R_{s} e\left(\bar{k}_{t}\right)\right\}
\end{aligned}
$$

Moreover, it could be obtained from Lemma 2 and Assumption 2 that

$$
\begin{gathered}
\varpi(k)^{T}\left[\begin{array}{cc}
-\Lambda L_{1} & \Lambda L_{2} \\
* & -\Lambda
\end{array}\right] \varpi(k) \geq 0 \\
\varpi(k)^{T}\left[\begin{array}{cc}
-\Gamma M_{1} & \Gamma M_{2} \\
* & -\Gamma
\end{array}\right] \varpi(k) \geq 0
\end{gathered}
$$

where $\varpi(k)=\left[\begin{array}{ll}e^{T}(k) & \tilde{f}^{T}(e(k))\end{array}\right]^{T}$. As such, it can be obtained according to inequalities (17)-(18) that

$$
\begin{aligned}
& \mathbb{E}\{(1-\gamma) \mathcal{V}(k)+\Delta \mathcal{V}(k)\} \\
= & \sum_{i=1}^{4} \mathbb{E}\left\{(1-\gamma) \mathcal{V}_{i}(k)+\Delta \mathcal{V}_{i}(k)\right\} \\
\leq & \sum_{i=1}^{4} \mathbb{E}\left\{(1-\gamma) \mathcal{V}_{i}(k)+\Delta \mathcal{V}_{i}(k)\right\} \\
& +\mathbb{E}\left\{\varpi(k)^{T}\left[\begin{array}{cc}
-\Lambda L_{1} & \Lambda L_{2} \\
* & -\Lambda
\end{array}\right] \varpi(k)\right\} \\
& +\mathbb{E}\left\{\varpi(k)^{T}\left[\begin{array}{cc}
-\Gamma M_{1} & \Gamma M_{2} \\
* & -\Gamma
\end{array}\right] \varpi(k)\right\} \\
\leq & \mathbb{E}\left\{\eta^{T}(k) \Phi \eta(k)\right\}+B^{T} P B+\sum_{s=1}^{l} D_{s}^{T} K_{s}^{T} P K_{s} D_{s}
\end{aligned}
$$

where

$$
\eta(k)=\left[\begin{array}{c}
\tilde{e}(k) \\
\tilde{f}(e(k)) \\
\tilde{g}(e(k)) \\
\tilde{g}(e(k-d(k)))
\end{array}\right], \quad \tilde{e}(k)=\left[\begin{array}{c}
e\left(\bar{k}_{0}\right) \\
e\left(\bar{k}_{1}\right) \\
\vdots \\
e\left(\overline{\bar{k}}_{\bar{\tau}}\right)
\end{array}\right] .
$$

Based on the condition (12), we have

$\mathbb{E}\{(1-\gamma) \mathcal{V}(k)+\Delta \mathcal{V}(k)\} \leq B^{T} P B+\sum_{s=1}^{l} D_{s}^{T} K_{s}^{T} P K_{s} D_{s}$ which implies that

$$
\begin{aligned}
\mathbb{E}\{\mathcal{V}(k)\} & \leq \mathbb{E}\left\{\gamma \mathcal{V}\left(\bar{k}_{1}\right)+\theta\right\} \\
& \leq \mathbb{E}\left\{\gamma^{2} \mathcal{V}\left(\bar{k}_{2}\right)+\gamma \theta+\theta\right\} \\
& \leq \mathbb{E}\left\{\gamma^{3} \mathcal{V}\left(\bar{k}_{3}\right)+\gamma^{2} \theta+\gamma \theta+\theta\right\} \\
& \leq \cdots \leq \mathbb{E}\left\{\gamma^{k} \mathcal{V}(0)+\frac{1-\gamma^{k}}{1-\gamma} \theta\right\} \\
& \leq \mathbb{E}\left\{\gamma^{k} \mathcal{V}(0)+\frac{1}{1-\gamma} \theta\right\}
\end{aligned}
$$

Let $\varepsilon_{*}$ be a prescribed upper bound of $\mathbb{E}\left\{\|\tilde{z}(k)\|^{2}\right\}$. Then, according to (14), there must exist a $0<\sigma<1$ satisfying

$$
\theta=\sigma \varepsilon_{*}(1-\gamma)
$$

Noticing Definition 1 and (13), we have

$$
\begin{aligned}
\mathbb{E}\left\{\|\tilde{z}(k)\|^{2}\right\} & =\mathbb{E}\left\{e^{T}(k) L^{T} L e(k)\right\} \\
& \leq \mathbb{E}\{\mathcal{V}(k)\} \\
& \leq \mathbb{E}\left\{\gamma^{k} \mathcal{V}(0)+\sigma \varepsilon_{*}\right\}
\end{aligned}
$$

which means that the SLT function $k_{*}$ can be derived as follows:

$$
k_{*}=\left\{\begin{array}{c}
0, \varepsilon_{*} \geq \frac{\mathbb{E}\{V(0)\}}{1-\sigma} \\
\left\lceil\log _{\gamma} \frac{(1-\sigma) \varepsilon_{*}}{\mathbb{E}\{V(0)\}}\right\rceil, \varepsilon_{*}<\frac{\mathbb{E}\{V(0)\}}{1-\sigma}
\end{array}\right.
$$

The proof is complete now.

By now, we have accomplished the analysis task in Theorem 1. Next, we are going to move onto the design issue of the estimator parameters $K_{i}(i=1,2, \cdots, l)$.

Theorem 2: Consider the estimator error dynamics (10). Let the positive scalar $0<\gamma<1$ and the desired upper bound $\varepsilon_{*}$ of SEE be given. Then, the dynamical system (10) is FTBMS if there exist $l+4$ PDMs $Q>0, P>0$, $R_{i}>0(1 \leq i \leq l), \Gamma \triangleq \operatorname{diag}\left(\gamma_{1}, \gamma_{2}, \cdots, \gamma_{n_{x}}\right)>0, \Lambda \triangleq$ $\operatorname{diag}\left(\lambda_{1}, \lambda_{2}, \cdots, \lambda_{n_{x}}\right)>0$, and $l$ matrices $Z_{1}, Z_{2}, \cdots, Z_{l}$ satisfying

$$
\begin{aligned}
\Xi & <0 \\
L^{T} L & \leq P \\
\Omega & <0
\end{aligned}
$$

where

$$
\begin{aligned}
& \bar{\Xi}=-P, \quad \Xi=\left[\begin{array}{cc}
\Xi_{11} & * \\
\Xi_{21} & \Xi_{22}
\end{array}\right], \quad \Xi_{11}=\left[\begin{array}{cc}
\tilde{\Sigma}_{11} & * \\
\tilde{\Sigma}_{21} & \tilde{\Sigma}_{22}
\end{array}\right] \text {, } \\
& \Xi_{21}=\left[\begin{array}{lllllll}
\tilde{\Xi}^{T} & \tilde{\Xi}_{0}^{T} & \ldots & \tilde{\Xi}_{\bar{\tau}}^{T} & \tilde{\Xi}_{1 l}^{T} & \ldots & \tilde{\Xi}_{l-1, l}^{T}
\end{array}\right]^{T} \text {, } \\
& \Xi_{22}=\operatorname{diag}\left\{\bar{\Xi}, \bar{\Xi}_{0}, \cdots, \bar{\Xi}_{\bar{\tau}}, \bar{\Xi}_{1 l}, \cdots, \bar{\Xi}_{l-1, l}\right\} \text {, } \\
& \tilde{\Sigma}_{11}=\left[\begin{array}{cccccc}
\beth_{00} & * & * & * & * & * \\
I_{10} & I_{11} & * & * & * & * \\
I_{20} & 0 & I_{22} & * & * & * \\
I_{30} & 0 & 0 & I_{33} & * & * \\
\vdots & \vdots & \vdots & \vdots & \ddots & * \\
\Pi_{\bar{\tau} 0} & 0 & 0 & 0 & \cdots & I_{\bar{\tau} \bar{\tau}}
\end{array}\right] \text {, } \\
& \tilde{\Sigma}_{21}=\left[\begin{array}{llll}
\beth_{\bar{\tau}_{1}, 0} & \beth_{\bar{\tau}_{1}, 1} & \ldots & \beth_{\bar{\tau}_{1}, \bar{\tau}} \\
\beth_{\bar{\tau}_{2}, 0} & \beth_{\bar{\tau}_{2}, 1} & \ldots & \beth_{\bar{\tau}_{2}, \bar{\tau}} \\
\beth_{\bar{\tau}_{3}, 0} & \beth_{\bar{\tau}_{3}, 1} & \cdots & \beth_{\bar{\tau}_{3}, \bar{\tau}}
\end{array}\right],
\end{aligned}
$$




$$
\begin{aligned}
& \tilde{\Sigma}_{22}=\left[\begin{array}{ccc}
\beth_{\bar{\tau}_{1}, \bar{\tau}_{1}} & * & * \\
\beth_{\bar{\tau}_{2}, \bar{\tau}_{1}} & \beth_{\bar{\tau}_{2}, \bar{\tau}_{2}} & * \\
\beth_{\bar{\tau}_{3}, \bar{\tau}_{1}} & \beth_{\bar{\tau}_{3}, \bar{\tau}_{2}} & \beth_{\bar{\tau}_{3}, \bar{\tau}_{3}}
\end{array}\right] \\
& I_{i j}=\left\{\begin{array}{l}
-\gamma P+\sum_{s=1}^{l} \bar{\tau} R_{s}-\Lambda L_{1}-\Gamma M_{1} \\
-\sum_{s=1}^{l} p_{s i} A^{T} Z_{s} C-\sum_{s=1}^{l} p_{s i} C^{T} Z_{s}^{T} A, i, j=0 \\
-\sum_{s=1}^{l} \gamma^{i} R_{s}, \quad i=j, i, j=1,2, \ldots, \bar{\tau} \\
-\sum_{s=1}^{l} p_{s i} C^{T} Z_{s}^{T} A, \quad j=0, i=1,2, \ldots, \bar{\tau}
\end{array}\right. \\
& I_{\bar{\tau}_{1}, i}=\left\{\begin{array}{l}
-\sum_{s=1}^{l} p_{s i} F^{T} Z_{s} C+\Lambda L_{2}, \quad i=0 \\
-\sum_{s=1}^{l} p_{s i} F^{T} Z_{s} C, i=1,2, \ldots, \bar{\tau} \\
-\Lambda, \quad i=\bar{\tau}+1
\end{array}\right. \\
& \beth_{\bar{\tau}_{2}, i}=\left\{\begin{array}{l}
\Gamma M_{2}, \quad i=0 \\
0, \quad 1 \leq i \leq \bar{\tau}_{1} \\
\left(d_{M}-d_{m}+1\right) Q-\Gamma, \quad i=\bar{\tau}_{2}
\end{array}\right. \\
& \boldsymbol{J}_{\bar{\tau}_{3}, i}=\left\{\begin{array}{l}
-\sum_{s=1}^{l} p_{s i} G^{T} Z_{s} C, \quad 1 \leq i \leq \bar{\tau} \\
0, \quad i=\bar{\tau}_{1}, \bar{\tau}_{2} \\
-Q, \quad i=\bar{\tau}_{3}
\end{array}\right. \\
& \tilde{\Xi}=\left[\begin{array}{lllllll}
P A & 0 & \cdots & 0 & P F & 0 & P G
\end{array}\right] \\
& \tilde{\Xi}_{0}=\left[\begin{array}{ccccccc}
\sqrt{p_{10}} Z_{1} C & 0 & \cdots & 0 & 0 & 0 & 0 \\
\sqrt{p_{20}} Z_{2} C & 0 & \cdots & 0 & 0 & 0 & 0 \\
\vdots & \vdots & \vdots & \vdots & \vdots & \vdots & \vdots \\
\sqrt{p_{l 0}} Z_{l} C & 0 & \cdots & 0 & 0 & 0 & 0
\end{array}\right], \\
& \bar{\Xi}_{0}=\operatorname{diag}_{l}\{-P\} \text {, } \\
& \tilde{\Xi}_{1}=\left[\begin{array}{ccccccc}
0 & \sqrt{p_{11}} Z_{1} C & \cdots & 0 & 0 & 0 & 0 \\
0 & \sqrt{p_{21}} Z_{2} C & \cdots & 0 & 0 & 0 & 0 \\
\vdots & \vdots & \vdots & \vdots & \vdots & \vdots & \vdots \\
0 & \sqrt{p_{l 1}} Z_{l} C & \cdots & 0 & 0 & 0 & 0
\end{array}\right] \\
& \bar{\Xi}_{1}=\operatorname{diag}_{l}\{-P\} \text {, }
\end{aligned}
$$$$
\tilde{\Xi}_{\bar{\tau}}=\left[\begin{array}{ccccccc}
0 & 0 & \cdots & \sqrt{p_{1 \bar{\tau}}} Z_{1} C & 0 & 0 & 0 \\
0 & 0 & \cdots & \sqrt{p_{2 \bar{\tau}}} Z_{2} C & 0 & 0 & 0 \\
\vdots & \vdots & \vdots & \vdots & \vdots & \vdots & \vdots \\
0 & 0 & \cdots & \sqrt{p_{l \bar{\tau}}} Z_{l} C & 0 & 0 & 0
\end{array}\right],
$$$$
\bar{\Xi}_{\bar{\tau}}=\operatorname{diag}_{l}\{-P\} \text {, }
$$$$
\tilde{\Xi}_{i l}=\left[\begin{array}{ccc}
p_{(i+1) 0} Z_{i+1} C & p_{(i+1) 1} Z_{i+1} C & \ldots \\
p_{i 0} Z_{i} C & p_{i 1} Z_{i} C & \ldots \\
p_{(i+2) 0} Z_{i+2} C & p_{(i+2) 1} Z_{i+2} C & \ldots \\
p_{i 0} Z_{i} C & p_{i 1} Z_{i} C & \ldots \\
\vdots & \vdots & \ddots \\
p_{l 0} Z_{l} C & p_{l 1} Z_{l} C & \cdots \\
p_{i 0} Z_{i} C & p_{i 1} Z_{i} C & \cdots
\end{array}\right.
$$$$
p_{(i+1) \bar{\tau}} Z_{i+1} C \quad 0 \quad 0 \quad 07
$$$$
p_{i \bar{\tau}} Z_{i} C \quad 0 \quad 0 \quad 0
$$$$
p_{(i+2) \bar{\tau}} Z_{i+2} C \quad 0 \quad 0 \quad 0
$$$$
p_{i \bar{\tau}} Z_{i} C \quad 0 \quad 0 \quad 0 \quad, \quad i=1,2, \cdots, l-1,
$$$$
\begin{array}{cccc}
\vdots & \vdots & \vdots & \vdots \\
p_{l \bar{\tau}} Z_{l} C & 0 & 0 & 0 \\
p_{i=} Z_{i} C & 0 & 0 & 0
\end{array}
$$$$
p_{i \bar{\tau}} Z_{i} C \quad 0 \quad 0 \quad 0
$$$$
\bar{\Xi}_{i l}=\operatorname{diag}_{2(l-i)}\{-P\}, \quad i=1,2, \cdots, l-1 \text {, }
$$

$$
\Omega=\left[\begin{array}{ccccc}
B^{T} P B-(1-\gamma) \varepsilon_{*} & * & * & * & * \\
Z_{1} D_{1} & -P & * & * & * \\
Z_{2} D_{2} & 0 & -P & * & * \\
\vdots & \vdots & \vdots & \ddots & \vdots \\
Z_{l} D_{l} & 0 & 0 & 0 & -P
\end{array}\right]
$$

Furthermore, if $\left(P, Q, R_{1}, R_{2}, \cdots, R_{l}, \Lambda, \Gamma, Z_{1}, Z_{2}, \cdots, Z_{l}\right)$ is a feasible solution of (20)-(22), then the parameters of the admissible finite-time SE can be acquired through matrices $Z_{i}(i=1,2, \cdots, l)$ as follows:

$$
K_{i}=P^{-1} Z_{i}, i=1,2, \cdots, l
$$

Proof: First, we denote

$$
\begin{aligned}
& \alpha_{i 0}=\left[\begin{array}{lllllll}
\sqrt{p_{i 0}} K_{i} C & 0 & \cdots & 0 & 0 & 0 & 0
\end{array}\right] \\
& \alpha_{i 1}=\left[\begin{array}{lllllll}
0 & \sqrt{p_{i 1}} K_{i} C & \cdots & 0 & 0 & 0 & 0
\end{array}\right] \\
& \alpha_{i \bar{\tau}}=\left[\begin{array}{lllllll}
0 & 0 & \cdots & \sqrt{p_{i \bar{\tau}}} K_{i} C & 0 & 0 & 0
\end{array}\right] \\
& i=1,2, \ldots, l \\
& \alpha_{j}=\left[\begin{array}{llll}
\alpha_{1 j}^{T} & \alpha_{2 j}^{T} & \cdots & \alpha_{l j}^{T}
\end{array}\right]^{T} \\
& j=0,1, \ldots, \bar{\tau} \\
& \beta_{i j}=\left[\begin{array}{ccccccc}
p_{j 0} K_{j} C & p_{j 1} K_{j} C & \cdots & p_{j \bar{\tau}} K_{j} C & 0 & 0 & 0 \\
p_{i 0} K_{i} C & p_{i 1} K_{i} C & \cdots & p_{i \bar{\tau}} K_{i} C & 0 & 0 & 0
\end{array}\right] \\
& 1 \leq i<j \leq l \\
& \beta_{i}=\left[\begin{array}{llll}
\beta_{i, i+1}^{T} & \beta_{i, i+2}^{T} & \cdots & \beta_{i, l}^{T}
\end{array}\right]^{T}, \quad 1 \leq i \leq l-1 \\
& \Theta=\left[\begin{array}{lllllll}
\tilde{\Xi}^{T} & \alpha_{0}^{T} & \cdots & \alpha_{\bar{\tau}}^{T} & \beta_{1}^{T} & \cdots & \beta_{l-1}^{T}
\end{array}\right] \\
& \varrho=l(l-1)+(\bar{\tau}+1) l+1 \\
& \Upsilon=\operatorname{diag}_{\varrho}\{P, P, \cdots, P\}
\end{aligned}
$$

Using Lemma 1 and applying the change of variables through $Z_{j}=P K_{j}(j=1,2, \cdots, l)$, it can be seen that $\Phi=\Xi_{11}+\Theta^{T} \Upsilon \Theta=\Xi_{11}-(\Upsilon \Theta)^{T}(-\Upsilon)^{-1}(\Upsilon \Theta)<0$ is guaranteed by the LMI (20). Note also that (14) can be rewritten as $\sum_{i=1}^{l} D_{i}^{T} K_{i}^{T} P K_{i} D_{i}+B^{T} P B-(1-\gamma) \varepsilon_{*}<0$, which is guaranteed by the LMI (22) from Lemma 1. According to Theorem 1, the SEE dynamics is FTBMS. The proof of this theorem is now complete.

Having designed the finite-time estimator, we are now going to aim at solving an optimization problem for the SEE dynamics (10), that is, we would like to minimize the SLT.

Theorem 3: Let the prescribed positive scalar $0<\gamma<1$, the desired upper bound $\varepsilon_{*}$ of SEE and the upper bound of $\|e(i)\|^{2}$ and $\|\tilde{g}(e(i))\|^{2}\left(i=0,-1, \cdots,-d_{M}\right), \vec{e}_{i}$ and $\tilde{g}\left(\vec{e}_{i}\right)$, be given. The SEE dynamics (10) is FTBMS if there exist $2 l+6$ PDMs $Q>0, P>0, R_{i}>0(1 \leq i \leq l), \Gamma=$ $\operatorname{diag}\left(\gamma_{1}, \gamma_{2}, \cdots, \gamma_{n_{x}}\right)>0, \Lambda=\operatorname{diag}\left(\lambda_{1}, \lambda_{2}, \cdots, \lambda_{n_{x}}\right)>0$, $S_{P}>0, S_{Q}>0, S_{R_{i}}>0(1 \leq i \leq l)$ and $l$ matrices $Z_{1}, Z_{2}$, $\cdots, Z_{l}$ such that the optimization problem

$$
\min _{\bar{X}, S_{\bar{X}}, \Lambda, \Gamma, Z_{1}, Z_{2}, \cdots, Z_{l}} \operatorname{trace}\left\{S_{P}+S_{Q}+\sum_{i=1}^{l} S_{R_{i}}\right\}
$$


with constraints (20), (21), (22) and constraints

$$
\begin{aligned}
& {\left[\begin{array}{cc}
-S_{P} & P^{T} \\
P & -I
\end{array}\right]<0,\left[\begin{array}{cc}
-S_{Q} & Q^{T} \\
Q & -I
\end{array}\right]<0,} \\
& {\left[\begin{array}{cc}
-S_{R_{i}} & R_{i}^{T} \\
R_{i} & -I
\end{array}\right]<0, i=1,2, \cdots, l}
\end{aligned}
$$

has feasible solution, where $\bar{X}$ and $S_{\bar{X}}$ denote, respectively, set $\left\{P, Q, R_{1}, R_{2}, \cdots, R_{l}\right\} \quad$ and set $\left\{S_{P}, S_{Q}, S_{R_{1}}, S_{R_{2}}, \cdots, S_{R_{l}}\right\}$. Furthermore, when (24) is feasible, the estimator parameters can be given by (23) and the upper bound of minimum SLT $k_{*}, \vec{k}_{*}$, can be calculated by

$$
\vec{k}_{*}=\log _{\gamma} \frac{(1-\sigma) \varepsilon_{*}}{\mathbb{E}\{\nu\}}
$$

where

$$
\begin{aligned}
\nu= & \mathbb{E}\left\{\|P\|_{F} \cdot \vec{e}_{0}\right. \\
& +\sum_{j=-d_{M}}^{-1} \gamma^{-1-j}\left(d_{M}-d_{m}+1\right)\|Q\|_{F} \cdot \tilde{g}\left(\vec{e}_{j}\right) \\
& \left.+\sum_{s=1}^{l} \sum_{t=1}^{\bar{\tau}} \sum_{j=-t}^{-1} \gamma^{-1-j}\left\|R_{s}\right\|_{F} \cdot \vec{e}_{j}\right\}
\end{aligned}
$$

Proof: According to Theorem 2, it can be proved that the dynamical system (10) is FTBMS and (23) is the explicit expression of the desired estimator parameters. Moreover, by using Lemma 1, constraint (25) is equivalent to $P^{T} P \leq S_{P}$, $Q^{T} Q \leq S_{Q}, R_{i}^{T} R_{i} \leq S_{R_{i}}(1 \leq i \leq l)$. Noticing the fact that, for a positive-definite matrix $P$ and a vector $x, x^{T} P x \leq$ $\|P\|_{F} \cdot\|x\|^{2}=\sqrt{\operatorname{trace}\left(P^{T} P\right)} \cdot\|x\|^{2}$ and the form of $\mathbb{E}\{\mathcal{V}(0)\}$, we can gain the conclusion that the optimization problem (24) could achieve the optimization of $\mathbb{E}\{\mathcal{V}(0)\}$. According to Theorem 1, the SLT function $k_{*}$ can be calculated by (15) and one can find that the smaller the $\mathbb{E}\{\mathcal{V}(0)\}$, the minimum the SLT $k_{*}$. This completes the proof of this theorem.

Remark 3: In the above theorem, we have proposed sufficient conditions to achieve the FTB of the SEE dynamics in the mean square with the optimized SLT function through an optimization problem with particular solution matrices. Moreover, the explicit expressions of estimator parameters for (9) and the optimized SLT have been given in the meanwhile.

Remark 4: In the past decades, fault detection and faulttolerant control have gained more and more research interest due to their obvious significance [19].In this paper, we have investigated the case that the probability distribution of transmission delay existing in each channel is known. Note that, the delay step considered is bounded, which means that the channel failure and sensor faults have not been considered here. One of our future research topics is to extended our main results to the finite-time state estimation problem subject to sensor faults or channel failure by adopting some adequate fault detection methods.

\section{NUMERICAL EXAMPLE}

We shall give an illustrative numerical simulation in this section to confirm the correctness and effectiveness of the proposed theorem. Consider the NNs (1) and the output model (4) with the following parameters:

$$
\begin{aligned}
A= & {\left[\begin{array}{ccc}
1 & 0 & 0 \\
0 & 0.28 & 0 \\
0 & 0 & 0.1
\end{array}\right], F=\left[\begin{array}{ccc}
0.1 & 0.05 & 0.03 \\
0.06 & 0.1 & 0.05 \\
0.05 & 0.1 & 0.08
\end{array}\right], } \\
G= & {\left[\begin{array}{lll}
0.03 & 0.07 & 0.1 \\
0.02 & 0.03 & 0.04 \\
0.06 & 0.02 & 0.04
\end{array}\right], B=\left[\begin{array}{c}
0.05 \\
0.02 \\
0.01
\end{array}\right], } \\
C= & {\left[\begin{array}{lll}
0.2 & 0.4 & 0.3 \\
0.25 & 0.3 & 0.2
\end{array}\right], L=\left[\begin{array}{ccc}
1.5 & 1 & 0.5 \\
0.9 & 1 & 2
\end{array}\right], } \\
D_{1}= & {\left[\begin{array}{l}
0.01 \\
0.02
\end{array}\right], D_{2}=\left[\begin{array}{l}
0.01 \\
0.02
\end{array}\right], D_{3}=\left[\begin{array}{c}
0.01 \\
0.02
\end{array}\right], } \\
J(k)= & {\left[\begin{array}{l}
0.08 \cos (k) \\
0.05 \sin (k) \\
0.06 \cos (k)
\end{array}\right], d(k)=\bmod \left(\frac{k}{2}\right) . }
\end{aligned}
$$

The activation function is chosen as

$$
\begin{gathered}
f(x(k))=\left[\begin{array}{ll}
\tanh \left(0.3 x_{1}(k)\right) & \tanh \left(0.1 x_{2}(k)\right) \\
\tanh \left(-0.2 x_{3}(k)\right)
\end{array}\right]^{T} \\
g(x(k))=\left[\tanh \left(0.2 x_{1}(k)\right) \quad \tanh \left(-0.7 x_{2}(k)\right)\right. \\
\left.\quad \tanh \left(0.5 x_{3}(k)\right)\right]^{T}
\end{gathered}
$$

and it can then be calculated that

$$
\begin{aligned}
& L_{1}=\operatorname{diag}\{-1,-1,-1\}, M_{1}=\operatorname{diag}\{-1,-1,-1\}, \\
& L_{2}=\left[\begin{array}{lll}
0 & 0 & 0 \\
0 & 0 & 0 \\
0 & 0 & 0
\end{array}\right], M_{2}=\left[\begin{array}{lll}
0 & 0 & 0 \\
0 & 0 & 0 \\
0 & 0 & 0
\end{array}\right] .
\end{aligned}
$$

Assume that there are three communication channels between the NN and estimator. The corresponding stochastic transmission delay $\tau_{i}(k)(i=1,2,3)$ satisfy the following probability distribution:

$$
\begin{aligned}
& \operatorname{Pr}\left\{\tau_{i}(k)=0\right\}=0.85, \quad \operatorname{Pr}\left\{\tau_{i}(k)=1\right\}=0.1, \\
& \operatorname{Pr}\left\{\tau_{i}(k)=2\right\}=0.05
\end{aligned}
$$

Let $\gamma=0.6$ and $\varepsilon_{*}=0.5$. According to the results in Theorem 3, we adopt the MATLAB LMI toolbox to cope with the optimization problem (24) whose solution matrices have prescribed particular structure with the LMI constraints (20), (21) and (22). Then, the estimator gain matrices $K_{1}, K_{2}$ and $K_{3}$ can be achieved as follows:

$$
\begin{aligned}
& K_{1}=\left[\begin{array}{cc}
-1.8618 & 2.7192 \\
0.0326 & 0.0298 \\
-0.0471 & 0.0789
\end{array}\right] \\
& K_{2}=\left[\begin{array}{cc}
-1.8618 & 2.7192 \\
0.0326 & 0.0298 \\
-0.0471 & 0.0789
\end{array}\right] \\
& K_{3}=\left[\begin{array}{cc}
-1.8618 & 2.7192 \\
0.0326 & 0.0298 \\
-0.0471 & 0.0789
\end{array}\right]
\end{aligned}
$$

The corresponding simulation results are given in Figs. 25, in which Figs. 2-3 plot the state trajectories of $z_{i}(k)$ 
$(i=1,2)$ and their estimations $\hat{z}_{i}(k)(i=1,2)$ with the initial condition $x(0)=\left[\begin{array}{lll}3 & -5 & 1.6\end{array}\right]^{T}, \hat{x}(0)=\left[\begin{array}{lll}0 & 0 & 0\end{array}\right]^{T}$, $\phi(i)=\hat{\phi}(i)=0(i<0)$. Fig. 4 shows the dynamical evolution of the SEE $\tilde{z}(k)$. Fig. 5 shows the Euclidean norm of the SEE $\tilde{z}(k)$ and the prescribed upper bound $\varepsilon_{*}$. The SLT function $k_{*}$ can be computed as $k_{*}=16$, and it is easy to see that the square of Euclidean norm of the SEE $\tilde{z}(k)$ stays below its upper bound $\varepsilon_{*}$ in Fig. 5. For comparison purposes, the influence of redundant delayed channels on estimation performance have been observed in TABLE I, where $N$ stands for the number of delayed channels and $k_{*}$ represents the SLT function. It could be found from this simulation that increasing the number of redundant delayed channels would help lead to the reduction of the SLT $k_{*}$, which proves the effectiveness of our proposed estimator design algorithm. The impact of the communication channel parameters on estimation performance is given in TABLE II, where

$$
\begin{aligned}
\text { ave } & \triangleq \frac{1}{\vec{N} \cdot \vec{M}} \sum_{i=1}^{\vec{N}} \sum_{j=1}^{\vec{M}}\|e(j)\|^{2} \\
\bar{t} & \triangleq \frac{1}{\vec{N}} \sum_{i=1}^{\vec{N}} t_{i}
\end{aligned}
$$

with $\vec{N}, \vec{M}$ and $t_{i}$ being respectively the number of the simulation trials, the step size and the running time of the $i$-th simulation trial. The transmission delay $\tau_{i}(k)(i=1,2,3)$ of three cases are shown as follows:

- case 1: $\operatorname{Pr}\left\{\tau_{i}(k)=0\right\}=0.85, \operatorname{Pr}\left\{\tau_{i}(k)=1\right\}=0.1$, $\operatorname{Pr}\left\{\tau_{i}(k)=2\right\}=0.05$

- case 2: $\operatorname{Pr}\left\{\tau_{i}(k)=0\right\}=0.9, \operatorname{Pr}\left\{\tau_{i}(k)=1\right\}=0.1$, $\operatorname{Pr}\left\{\tau_{i}(k)=2\right\}=0$

- case 3: $\operatorname{Pr}\left\{\tau_{i}(k)=0\right\}=0.95, \operatorname{Pr}\left\{\tau_{i}(k)=1\right\}=0.025$, $\operatorname{Pr}\left\{\tau_{i}(k)=2\right\}=0.025$

Through TABLE II, the effectiveness of our proposed design method has been further proved.

TABLE I: The relationship between the number $N$ of redundant delayed channels and the upper bound $\vec{k}_{*}$ of SLT function $k_{*}$

\begin{tabular}{|c|c|}
\hline$N$ & $\vec{k}_{*}$ \\
\hline 3 & 16.4847 \\
4 & 13.8552 \\
5 & 13.6816 \\
\hline
\end{tabular}

TABLE II: The influence of the communication channels' parameters on estimation performance

\begin{tabular}{|c|c|c|c|}
\hline performance index & $\vec{k}_{*}$ & ave & $\bar{t}$ \\
\hline case 1 & 16.4847 & 0.0642 & 8.1180 \\
case 2 & 13.7452 & 0.0543 & 1.7856 \\
case 3 & 13.2471 & 0.0465 & 3.1821 \\
\hline
\end{tabular}

\section{CONCLUSION}

The FTSE problem has been addressed in this work for a type of delayed NNs subject to redundant delayed channels.

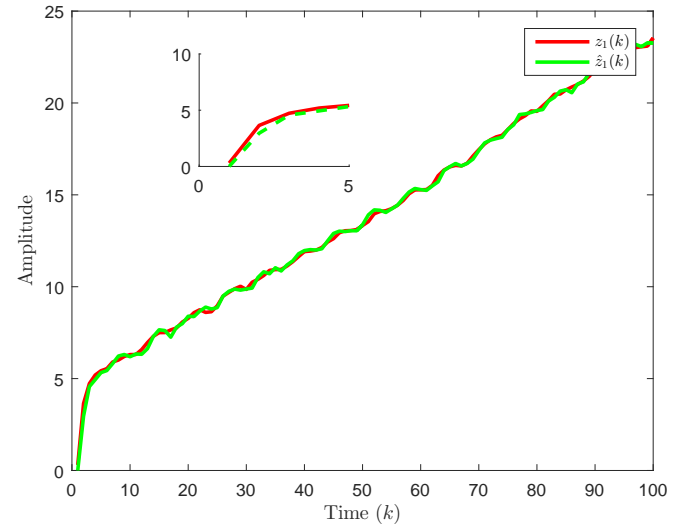

Fig. 2: The state evolutions of $z_{1}(k)$ and $\hat{z}_{1}(k)$.

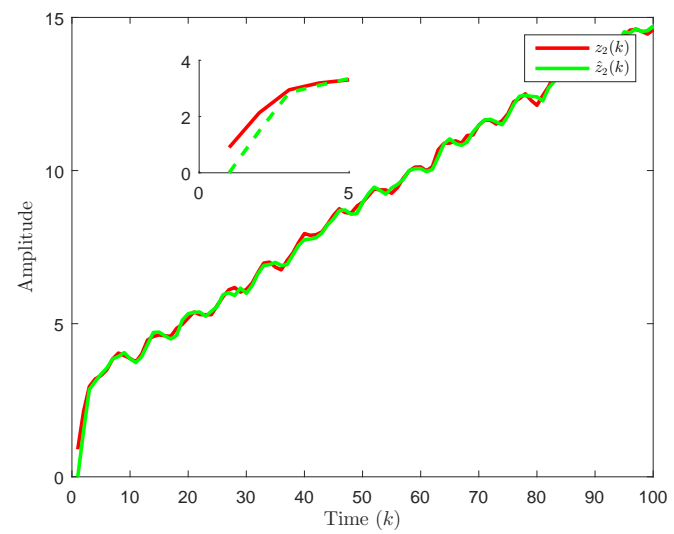

Fig. 3: The state evolutions of $z_{2}(k)$ and $\hat{z}_{2}(k)$.

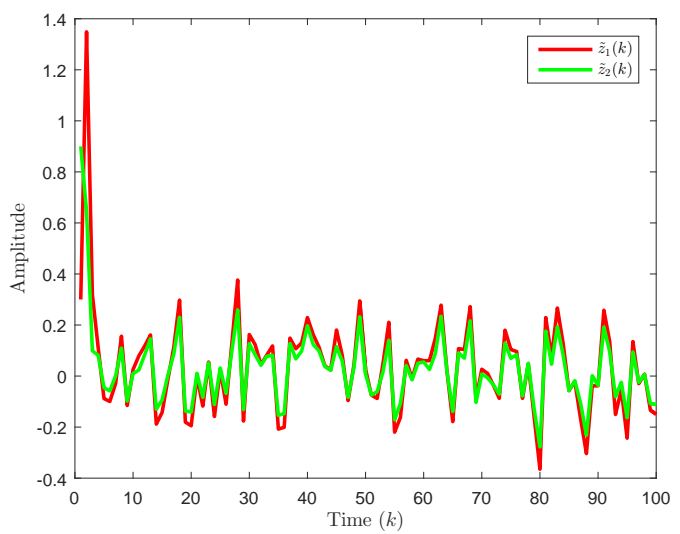

Fig. 4: The components $\tilde{z}_{i}(k)(i=1,2)$.

To improve communication performance, redundant channels have been used to design the finite-time SE with novel structure and the time delay phenomena existing in redundant channels have been concerned. By introducing a special Lyapunov-like functional corresponding to SEE dynamics and using the stochastic analysis technology, we have achieved sufficient conditions ensuring that the SEE dynamics actualizes FTBMS. Then, the desired estimator gains have been given by solving a special constrained optimization problem. Finally, a 


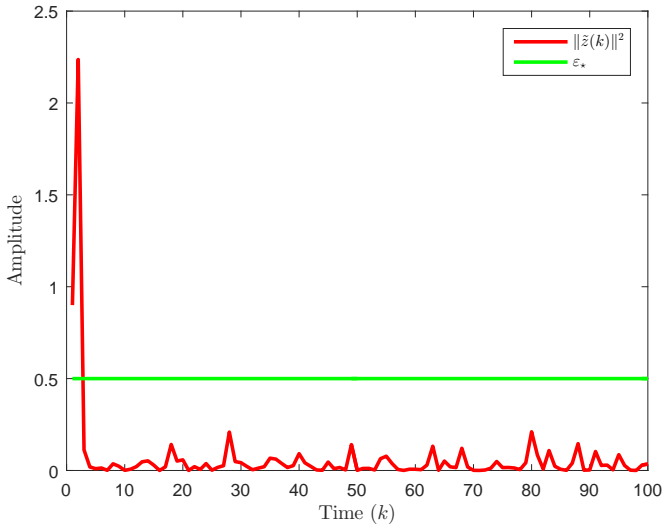

Fig. 5: The size of SEE $\tilde{z}(k)$ and its upper bound $\varepsilon_{\star}$.

numerical simulation has been adopted to show the correctness and the effectiveness of the developed design method of finitetime estimator. Further research topics include the $\mathcal{H}_{\infty}$ control problem and $\mathcal{H}_{\infty}$ filtering problem with redundant delayed channels/protocols [9], [10], [26], [32], [46]-[48]

\section{REFERENCES}

[1] M. Bahreini and J. Zarei, "Static-output feedback controller design for a class of nonlinear networked control systems with random delay and data packet dropout", Systems Science and Control Engineering: An Open Access Journal, vol. 4, no. 1, pp. 192-198, Aug. 2016.

[2] X. Bai, Z. Wang, L. Zou and F. E. Alsaadi, Collaborative fusion estimation over wireless sensor networks for monitoring $\mathrm{CO}_{2}$ concentration in a greenhouse, Information Fusion, vol. 42, pp. 119-126, Jul. 2018.

[3] G. Bao and Z. Zeng, "Region stability analysis for switched discrete-time recurrent neural network with multiple equilibria," Neurocomputing, vol. 249, pp. 182-190, Aug. 2017.

[4] S. Boyd, L. E. Ghaoul, E. Feron and V. Balakrishnan, "Linear matrix inequalities in systems and control theory," Society for industrial and applied mathematics, 1994.

[5] J. Cao, Z. Wu, J. Wu, and H. Xiong, SAIL: Summation-based incremental learning information-theoretic text clustering, IEEE Transactions on Cybernetics, vol. 43, no. 2, pp. 570-584, 2013. J. Cao, B. Wang, and B. Douglas, Similarity based leaf image retrieval using multiscale Rangle description, Information Sciences, vol. 374, pp. 51-64, 2016.

[6] J. Cheng, H. Zhu, S. Zhong, Y. Zhang and Y. Li, "Finite-time $\mathcal{H}_{\infty}$ control for a class of discrete-time Markovian jump systems with partly unknown time-varying transition probabilities subject to average dwell time switching," International Journal of Systems Science, vol. 46, no. 6 , pp. 1080-1093, Apr. 2015.

[7] X. Ding, J. Cao, A. Alsaedi, F. Alsaadi and T. Hayat, "Robust fixedtime synchronization for uncertain complex-valued neural networks with discontinuous activation functions," Neural Networks, vol. 90, pp. 42-55, June. 2017.

[8] D. Ding, Z. Wang, B. Shen and H. Dong, " $\mathcal{H}_{\infty}$ state estimation with fading measurements, randomly varying nonlinearities and probabilistic distributed delays," International Journal of Robust and Nonlinear Control, vol. 25, no. 13, pp. 2180-2195, Jun. 2015.

[9] D. Ding, Z. Wang, B. Shen and H. Dong, "Envelope-constrained $\mathcal{H}_{\infty}$ filtering with fading measurements and randomly occurring nonlinearities: the finite horizon case," Automatica, vol. 55, pp. 37-45, May 2015.

[10] H. Dong, Z. Wang, S. X. Ding and H. Gao, "On $\mathcal{H}_{\infty}$ Estimation of Randomly Occurring Faults for A Class of Nonlinear Time-Varying Systems With Fading Channels," IEEE Transactions on Automatic Control, vol. 64, no. 2, Feb. 2016.

[11] W. Gao and R. R. Selmic, "Neural network control of a class of nonlinear systems with actuator saturation," IEEE Transactions on Neural Networks, vol. 17, no. 1, pp. 147-156, Jan. 2006.

[12] Y. He, Q. Wang, M. Wu and C. Lin, "Delay-dependent state estimation for delayed neural networks," IEEE Transactions on Neural Networks, vol. 17, no. 4, pp. 1077-1081, Jul. 2006
[13] J. Hu, Z. Wang, H. Gao and L. K. Stergioulas, "Extended Kalman filtering with stochastic nonlinearities and multiple missing measurements," Automatica, vol. 48, no. 9, pp. 2007-2015, Sept. 2012.

[14] S. Hu, D. Yue and J. Liu, " $\mathcal{H}_{\infty}$ filtering for networked systems with partly known distribution transmission delays," Information Sciences, vol. 194, pp. 270-282, Jul. 2012.

[15] G. Joya, M. Atencia and F. Sandoval, "Hopfield neural networks for optimization: study of the different dynamics," Neurocomputing, vol. 43, pp. 219-237, Mar. 2002.

[16] H. R. Karimi and H. Gao, "New delay-dependent exponential $\mathcal{H}_{\infty}$ synchronization for uncertain neural networks with mixed time delays," IEEE Transactions on Systems, Man, and Cybernetics-Part B: Cybernetics, vol. 40, no. 1, pp. 173-185, Feb. 2010.

[17] J. Li, H. Dong, F. Han, N. Hou and X. Li, "Filter design, fault estimation and reliable control for networked time-varying systems: a survey", Systems Science and Control Engineering, vol. 5, no. 1, pp. 331-341, Jul. 2017.

[18] W. Li, G. Wei, D. Ding, Y. Liu and F. E. Alsaadi, "A new look at boundedness of error covariance of Kalman filtering", IEEE Transactions on Systems, Man, and Cybernetics: Systems, vol. 48, no. 2, pp. 309-314, Feb. 2018

[19] Y. Li, H. R. Karimi, Q. Zhang, D. Zhao and Y. Li, "Fault detection for linear discrete time-varying systems subject to random sensor delay: a Riccati equation approach," IEEE Transactions on Circuits and SystemsI:Regular Papers, vol. 65, no. 5, May. 2018.

[20] H. Liu, Z. Wang, B. Shen and X. Liu, Event-triggered $H_{\infty}$ state estimation for delayed stochastic memristive neural networks with missing measurements: The discrete time case, IEEE Transactions on Neural Networks and Learning Systems, vol. 29, no. 8, pp. 3726-3737, Aug. 2018.

[21] H. Liu, Z. Wang, B. Shen, T. Huang and F. E. Alsaadi, Stability analysis for discrete-time stochastic memristive neural networks with both leakage and probabilistic delays, Neural Networks, vol. 102, pp. 1-9, Jun. 2018.

[22] P. Liu, Z. Zeng and J. Wang, "Multistability of recurrent neural networks with nonmonotonic activation functions and mixed time delays", IEEE Transactions on Systems, Man, and Cybernetics: Systems, vol. 46, no. 4, pp. 512-523, Apr. 2016.

[23] Y. Liu, Z. Wang and J. Liang, "Stability and synchronization of discretetime Markovian jumping neural networks with mixed mode-dependent time delays," IEEE Transactions on Neural Networks, vol. 20, no. 7, pp. 1102-1116, Jul. 2009.

[24] Y. Liu, Z. Wang, J. Liang and X. Liu, "Synchronization of coupled neutral-type neural networks with jumping-mode-dependent discrete and unbounded distributed delays," IEEE Transactions on Cybernetics, vol. 43, no. 1, pp. 102-114, Feb. 2013.

[25] Y. Liu, Z. Wang and X. Liu, "Global exponential stability of generalized recurrent neural networks with discrete and distributed delays," Neural Networks, vol. 19, pp. 667-675, Jun. 2006.

[26] Y. Luo, Z. Wang and G. Wei, Fault detection for fuzzy systems with multiplicative noises under periodic communication protocols, IEEE Transactions on Fuzzy Systems, Vol. 26, No. 4, pp. 2384-2395, Aug. 2018.

[27] H. Qiao, J. Peng, Z. B. Xu and B. Zhang, "A reference model approach to stability analysis of neural networks," IEEE Transactions on Systems, Man, and Cybernetics, Part B(Cybernetics), vol. 33, no. 6, pp. 925-936, Dec. 2003.

[28] M. Seera, C. Lim, K. Tan and W. Liew, "Classification of transcranial Doppler signals using individual and ensemble recurrent neural networks," Neurocomputing, vol. 249, pp. 337-344, Aug. 2017.

[29] B. Shen, Z. Wang and H. Tan, Guaranteed cost control for uncertain nonlinear systems with mixed time-delays: The discrete-time case, European Journal of Control, Vol. 40, pp. 62-67, Mar. 2018.

[30] B. Shen, Z. Wang and H. Qiao, Event-triggered state estimation for discrete-time multidelayed neural networks with stochastic parameters and incomplete measurements, IEEE Transactions on Neural Networks and Learning Systems, Vol. 28, No. 5, pp. 1152-1163, May 2017.

[31] W. Shen, Z. Zeng and S. Wen, "Synchronization of complex dynamical network with piecewise constant argument of generalized type," Neurocomputing, vol. 173, pp. 671-675, Jan. 2016.

[32] L. Sheng, W. Zhang and M. Gao, "Relationship between Nash equilibrium strategies and $\mathcal{H}_{2} / \mathcal{H}_{\infty}$ control of stochastic Markov jump systems with multiplicative noise", IEEE Transactions on Automatic Control, vol. 59, no. 9, pp. 2592-2597, Sep. 2014.

[33] J. Wang, H. Wu, T. Huang, S. Ren and J. Wu, "Passivity analysis of coupled reaction-diffusion neural networks with Dirichlet bounded 
This article has been accepted for publication in a future issue of this journal, but has not been fully edited. Content may change prior to final publication. Citation information: DOI10.1109/TSMC.2018.2874508, IIEEE Transactions on Systems, Man, and Cybernetics: Systems

conditions", IEEE Transactions on Systems, Man and Cybernetics: Systems, vol. 47, no. 8, pp. 2148-2159, Aug. 2017.

[34] L. Wang, Z. Wang, G, Wei and F. Alsaadi, "Finite-Time State Estimation for Recurrent Delayed Neural Networks With Component-Based Event-Triggering Protocol," IEEE Transactions on Neural Networks and Learning Systems, vol. 29, vo. 4, pp. 1046-1057, Apr. 2018.

[35] L. Wang, Z. Wang, Q.-L. Han and G. Wei, Event-based varianceconstrained $H_{\infty}$ filtering for stochastic parameter systems over sensor networks with successive missing measurements, IEEE Transactions on Cybernetics, Vol. 48, No. 3, pp. 1007-1017, Mar. 2018.

[36] S. Wang, M. Zeng, J. Park and L. Zhang, "Finite-time control for networked switched linear systems with an event-driven communication approach," International Journal of Systems Science, vol. 48, no. 2, pp. 236-246, 2017.

[37] G. Wei, S. Liu, L. Wang and Y. Wang, "Event-based distributed setmembership filtering for a class of time-varying non-linear systems over sensor networks with saturation effects," International Journal of General Systems, vol. 45, no. 5, pp. 532-547, Apr. 2016.

[38] J. Wu, J. Li, G. Zong and W. Chen, "Global finite-time adaptive stabilization of nonlinearly parametrized systems with multiple unknown control directions", IEEE Transactions on Systems, Man and Cybernetics: Systems, vol. 47, no. 7, pp. 1405-1414, Jul. 2017.

[39] J. Xia, J. Park, H. Zeng and H. Shen, "Delay-difference-dependent robust exponential stability for uncertain stochastic neural networks with multiple delays," Neurocomputing, vol. 140, pp. 210-218, Sep. 2014.

[40] Q. Xiao and Z. Zeng, "Scale-Limited Lagrange Stability and Finite-Time Synchronization for Memristive Recurrent Neural Networks on Time Scales," IEEE Transactions on Cybernetics, vol. 47, no. 10, pp. 2984 2994, Oct. 2017.

[41] L. Yan, S. Zhang, D. Ding, Y. Liu and F. Alsaadi, " $\mathcal{H}_{\infty}$ state estimation for memristive neural networks with multiple fading measurements," Neurocomputing, vol. 230, pp. 23-29, Mar. 2017.

[42] F. Yang and Y. Li, "Set-membership filtering for systems with sensor saturation," Automatica, vol. 45, pp. 1896-1902, Aug. 2009.

[43] S. Zhang, D. Ding, G. Wei, Y. Liu and F. E. Alsaadi, "H(H) state estimation for artificial neural networks over redundant channels," Neurocomputing, vol. 226, pp. 117-125, Feb. 2017.

[44] Z. Zhao, Z. Wang, L. Zou and X. Bai, Finite-horizon $H_{\infty}$ state estimation for artificial neural networks with component-based distributed delays and stochastic protocol, Neurocomputing, in press, 2018, DOI: 10.1016/j.neucom.2018.08.031

[45] Y. Zhu, L. Zhang and W. Zheng, "Distributed $\mathcal{H}_{\infty}$ filtering for a class of discrete-time Markov jump Lur'e systems with redundant channels," IEEE Transactions on Industrial Electronics, vol. 63, no. 3, pp. 18761885, Mar. 2016.

[46] L. Zou, Z. Wang, Q.-L. Han and D. H. Zhou, "Recursive filtering for time-varying systems with random access protocol," IEEE Transactions on Automatic Control, in press. DOI: 10.1109/TAC.2018.2833154

[47] L. Zou, Z. Wang, Q.-L. Han and D. H. Zhou, "Ultimate boundedness control for networked systems with Try-Once-Discard protocol and uniform quantization effects," IEEE Transactions on Automatic Control, vol. 62, no. 12, pp. 6582-6588, Dec. 2017.

[48] L. Zou, Z. Wang, J. Hu and H. Gao, "On $\mathcal{H}_{\infty}$ finite-horizon filtering under stochastic protocol: dealing with high-rate communication networks," IEEE Transactions on Automatic Control, vol. 62, no. 9, pp. 4884-4890, Sept. 2017.

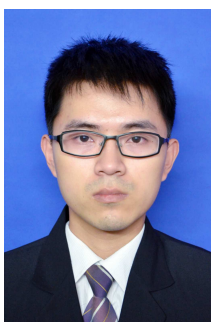

Zhongyi Zhao received the B.Eng. degree in electrical engineering and automation from Shandong University of Science and Technology, Qingdao, China, in 2016. He is currently pursuing the Ph.D. degree from the College of Electrical Engineering and Automation, Shandong University of Science and Technology, Qingdao, China. His current research interests include the control and filtering for networked system.

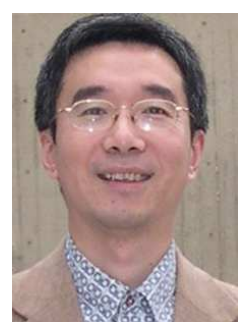

Zidong Wang (SM'03-F'14) was born in Jiangsu, China, in 1966. He received the B.Sc. degree in mathematics in 1986 from Suzhou University, Suzhou, China, and the M.Sc. degree in applied mathematics in 1990 and the Ph.D. degree in electrical engineering in 1994, both from Nanjing University of Science and Technology, Nanjing, China.

$\mathrm{He}$ is currently a Professor of Dynamical Systems and Computing in the Department of Computer Science, Brunel University London, U.K. From 1990 to 2002, he held teaching and research appointments in universities in China, Germany and the UK. Prof. Wang's research interests include dynamical systems, signal processing, bioinformatics, control theory and applications. He has published more than 400 papers in refereed international journals. He is a holder of the Alexander von Humboldt Research Fellowship of Germany, the JSPS Research Fellowship of Japan, William Mong Visiting Research Fellowship of Hong Kong.

Prof. Wang serves (or has served) as the Editor-in-Chief for Neurocomputing, Deputy Editor-in-Chief for International Journal of Systems Science, and an Associate Editor for 12 international journals including IEEE Transactions on Automatic Control, IEEE Transactions on Control Systems Technology, IEEE Transactions on Neural Networks, IEEE Transactions on Signal Processing, and IEEE Transactions on Systems, Man, and Cybernetics-Part C. $\mathrm{He}$ is a Fellow of the IEEE, a Fellow of the Royal Statistical Society and a member of program committee for many international conferences.

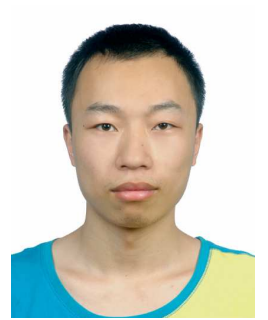

Lei Zou received the B.Sc. degree in automation from Beijing Institute of Petrochemical Technology, Beijing, China, in 2008, the M.Sc. degree in control science and engineering from China University of Petroleum (Beijing Campus), Beijing, China, in 2011 and the Ph.D degree in control science and engineering in 2016 from Harbin Institute of Technology, Harbin, China. From October 2013 to October 2015, he was a visiting Ph.D. student with the Department of Computer Science, Brunel University London, Uxbridge, U.K. He is currently a lecturer with the college of electrical engineering and automation, Shandong University of Science and Technology, Qingdao, China. His research interests include nonlinear stochastic control and filtering, as well as networked control under various communication protocols. He is a very active reviewer for many international journals.

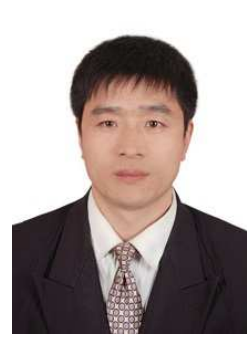

Ge Guo (SM'14) received the B.S. degree and the $\mathrm{PhD}$ degree from Northeastern University, Shenyang, China, in 1994 and 1998, respectively. In 1999 he joined Lanzhou University of Technology, Lanzhou, China, where he was the Director of the Institute of Intelligent Control and Robots and a Professor from July 2004 to May 2005. He then worked in Dalian Maritime University, Dalian, China, as a Professor with the Department of Automation. $\mathrm{He}$ is now a professor in Northeastern University. From 2009 to 2015 he also held a visiting research position at The Chinese University of Hong Kong. He has published over 100 journal papers within his areas of interest, which include intelligent transportation systems and cyber-physical systems. Dr. Guo is the Managing Editor of International Journal of Systems, Control and Communications and an Associate Editor of several other journals like Information Sciences, IEEE Intelligent Transportation Systems Magazine, and ACTA Automatica Sinica. He was an honoree of the New Century Excellent Talents in University, Ministry of Education, China, in 2004 and a nominee for Gansu Top Ten Excellent Youths by the Gansu Provincial Government in 2005. He was the CAA Young Scientist Award winner in 2017. 